OPEN ACCESS

Edited by: lda Karlsson,

Karolinska Institutet (KI), Sweden

Reviewed by:

William K. Scott,

University of Miami, United States

Giuseppe Passarino,

University of Calabria, Italy

*Correspondence:

Jennifer R. Dungan

jrdungan@ufl.edu

Specialty section: This article was submitted to

Genetics of Aging,

a section of the journal

Frontiers in Genetics

Received: 30 January 2021 Accepted: 04 May 2021

Published: 01 June 2021

Citation:

Dungan JR, Qin X, Hurdle M, Haynes CS, Hauser ER and Kraus WE

(2021) Genome-Wide Variants Associated With Longitudinal Survival Outcomes Among Individuals With

Coronary Artery Disease.

Front. Genet. 12:661497.

doi: 10.3389/fgene.2021.661497

\section{Genome-Wide Variants Associated With Longitudinal Survival Outcomes Among Individuals With Coronary Artery Disease}

\author{
Jennifer R. Dungan 1*, Xue Qin², Melissa Hurdle², Carol S. Haynes², \\ Elizabeth R. Hauser ${ }^{2,3,4}$ and William E. Kraus ${ }^{2,5}$
}

1 Division of Healthcare in Adult Populations, School of Nursing, Duke University, Durham, NC, United States, ${ }^{2}$ School of Medicine, Duke Molecular Physiology Institute, Duke University, Durham, NC, United States, ${ }^{3}$ Department of Biostatistics and Bioinformatics, Duke University School of Medicine, Durham, NC, United States, ${ }^{4}$ Cooperative Studies Program Epidemiology Center, Durham VA Medical Center, Durham, NC, United States, ${ }^{5}$ Division of Cardiology, Department of Medicine, School of Medicine, Duke University, Durham, NC, United States

Objective: Coronary artery disease (CAD) is an age-associated condition that greatly increases the risk of mortality. The purpose of this study was to identify gene variants associated with all-cause mortality among individuals with clinically phenotyped CAD using a genome-wide screening approach.

Approach and Results: We performed discovery $(n=684)$, replication $(n=404)$, and meta-analyses $(n=1,088)$ for association of genomic variants with survival outcome using secondary data from White participants with CAD from two GWAS sub-studies of the Duke Catheterization Genetics Biorepository. We modeled time from catheterization to death or last follow-up (median 7.1 years, max 12 years) using Cox multivariable regression analysis. Target statistical screening thresholds were $p \times$ $10^{-8}$ for the discovery phase and Bonferroni-calculated $p$-values for the replication $\left(p<5.3 \times 10^{-4}\right)$ and meta-analysis $\left(p<1.6 \times 10^{-3}\right)$ phases. Genome-wide analysis of 785,945 autosomal SNPs revealed two SNPs (rs13007553 and rs587936) that had the same direction of effect across all three phases of the analysis, with suggestive $p$-value association in discovery and replication and significant meta-analysis association in models adjusted for clinical covariates. The rs13007553 SNP variant, LINC01250, which resides between MYTIL and EIPR1, conferred increased risk for allcause mortality even after controlling for clinical covariates [HR 1.47, 95\% Cl 1.17-1.86, $p($ adj $)=1.07 \times 10^{-3}$ (discovery), $p($ adj $)=0.03$ (replication), $p($ adj $)=9.53 \times 10^{-5}$ (meta-analysis)]. MYT1L is involved in neuronal differentiation. TSSC1 is involved in endosomal recycling and is implicated in breast cancer. The rs587936 variant annotated to DAB2IP was associated with increased survival time [HR 0.65, 95\% $\mathrm{Cl} 0.51-0.83$, $p($ adj $)=4.79 \times 10^{-4}$ (discovery), $p($ adj $)=0.02$ (replication), $p($ adj $)=2.25 \times 10^{-5}$ (meta-analysis)]. DAB2IP is a ras/GAP tumor suppressor gene which is highly expressed in vascular tissue. DAB2IP has multiple lines of evidence for protection against atherosclerosis. 
Conclusion: Replicated findings identified two candidate genes for further study regarding association with survival in high-risk CAD patients: novel loci LINC01250 (rs13007553) and biologically relevant candidate DAB2IP (rs587936). These candidates did not overlap with validated longevity candidate genes. Future research could further define the role of common variants in survival outcomes for people with $C A D$ and, ultimately, improve longitudinal outcomes for these patients.

Keywords: coronary artery disease, survival analysis, genome-wide association study, age-related disease, candidate gene analyses

\section{INTRODUCTION}

Worldwide, more people die from heart disease than from any other cause (Virani et al., 2020). Up to half of the variation in CAD etiology has been attributed to genetic influences. In a 36year observational study of nearly 21,000 Swedish individuals who were twins, researchers estimated the heritability of risk of death from CAD to be $38-57 \%$ (Zdravkovic et al., 2002). Investigators in a Danish twin study achieved similar results, estimating the heritability of CHD mortality at $55 \%$ even after controlling for smoking and BMI (Wienke et al., 2005). Taken together, these results suggest that genetic factors are significant contributors to the risk of death from CAD.

Researchers have tested a limited number of CAD candidate genes for associations with mortality endpoints, with inconsistent findings. Candidates such as ADAM33, AGT, AGE, and ILRL1 have only been tested with short-term endpoints, showing variable effects and remaining unreplicated (GioliPereira et al., 2012; Ellis et al., 2013; Figarska et al., 2013; Patel et al., 2019). Variants in the well-studied 9p21 and 6p24 CAD candidate loci have also demonstrated sexand age-associated effects on risk of cardiac death (Ivanova et al., 2017). Previously, we identified a genetic component of survival in the context of clinically significant CAD-a novel phenotype we characterized as "survivorship with CAD" (Dungan et al., 2013). In that study, we conceptualized the genetic contribution to survivorship with $\mathrm{CAD}$ as age and phenotype dependent and as likely to share genetic variation with the lifespan longevity phenotype. Using this framework, we tested known CAD candidate genes for association with survival outcomes specifically in patients with prevalent CAD. We identified SNPs in LSAMP that had varied allelic effects on hazards of all-cause mortality, with some alleles conferring significantly increased hazard of all-cause mortality and others significantly improved likelihood of survival. Notably, these observed allelic effects were specific to CAD cases, having

Abbreviations: AIP1, apoptosis signal regulating kinase 1; ASK1, apoptosis signal-regulating kinase 1; BMI, body mass index; CABG, coronary artery bypass graft; CAD, coronary artery disease; CATHGEN, catheterization genetics biorepository; CHD, coronary heart disease; CI, confidence interval; DAB2IP, disabled homolog 2 interacting protein; DNA, deoxyribonucleic acid; EARP, endosome-associated recycling protein; EIPR1, EARP complex and GARP complex interacting protein 1; GWAS, genome-wide association study; HR, hazard ratio; $L S A M P$, limbic system-associated membrane protein; MAF, minor allele frequency; MI, myocardial infarction; MYT1L, myelin transcription factor 1like; Ras/GAP, ras/GTPase activating protein; SNP, single nucleotide polymorphism; TSSC1, tumor-suppressing subtransferable fragment candidate gene 1 . shown no significant association among non-CAD controls (Dungan et al., 2016). Following up on our prior candidategene results with an agnostic, genome-wide association analysis is an appropriate next step in the search for genomic variation that contributes to long-term survival outcomes among people with CAD.

The purpose of this study, then, was to perform a genomewide association screen for variants associated with survival among people with $\mathrm{CAD}$ to inform future hypothesis-driven work. We include replication and meta-analyses.

\section{MATERIALS AND METHODS}

\section{Design}

We conducted a secondary analysis of existing data from two separate GWAS sub-studies of participants sampled from the Catheterization Genetics study clinical cardiovascular biorepository (CATHGEN; $N=$ 9,334; Sutton et al., 2008; Kraus et al., 2015a,b). We employed a two-step genome-wide association screen for variants associated with survival outcomes in patients with CAD, using the separate GWAS sub-studies for discovery $(N=684)$ and replication $(N=404)$.

\section{Study Population}

The Duke University Institutional Review Board approved the primary CATHGEN cohort biorepository, GWAS sub-studies and the present GWA screen of survival in CAD. Briefly, patients were referred to CATHGEN from cardiac catheterization laboratories at Duke University (southeastern United States), where they were being evaluated for ischemic heart disease. Patients with severe pulmonary hypertension or transplant were ineligible for CATHGEN participation. All participants provided informed, written consent for participation in CATHGEN at the time of enrollment, which included DNA collection, medical record abstraction, and annual follow-up for mortality events. CATHGEN primary investigators drew samples from the primary CATHGEN cohort biorepository for the two GWAS sub-studies we evaluated in the present secondary analysis (gray boxes, Figure 1). The first sample was a sequential series of 2,023 CATHGEN participants $>18$ years of age whose primary reason for catheterization was concern for ischemic heart disease with complete coronary angiogram (Figure 1, gray box, left; Kraus et al., 2015b). The other sub-study sample $(N=1,490)$ consisted of a CAD case-control set of the same age and clinical 


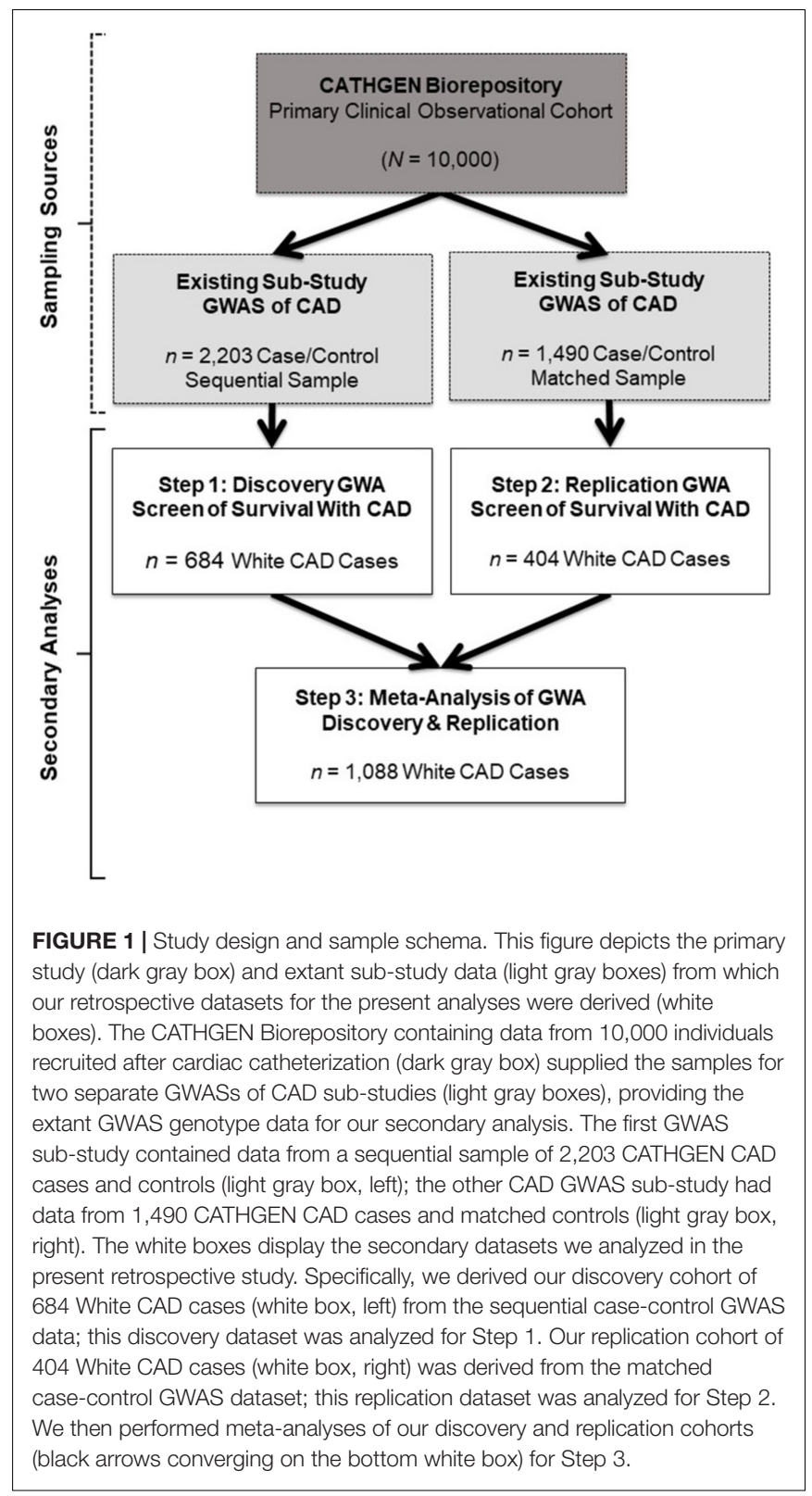

indication, matched to controls on age, race, and sex (745 CAD cases and 745 non-CAD controls; Figure 1 gray box, right; Kraus et al., 2015b).

\section{Inclusion Criteria}

We further applied the following criteria to the existing GWAS sub-study cohorts for inclusion in the present study's analyses. We included only self-reported White participants for two reasons: First, the frequency of self-reported Black/African American individuals with CAD in the extant GWAS datasets was insufficient for stratified analyses $(n=126$ and 124 for the discovery and replication cohorts, respectively). Second, our goal is for the present GWA screen to be comparable to the findings of our prior work as we look to build genomic convergence for this phenotype, and the sample for our earlier candidate gene study of survivorship with CAD comprised White individuals (Dungan et al., 2016).

We selected CAD-defined cases from the larger of the two sub-studies to serve as our GWAS discovery dataset $(n=684)$. As in the primary CATHGEN study, we defined positive CAD case status as having a Duke CAD index $\geq 32$ (at least one vessel having at least $75 \%$ stenosis) determined by clinical coronary heart catheterization (Sutton et al., 2008). Of note, the Duke CAD index reflects both the extent and location of stenosis. It is used as an indicator of disease severity and includes individuals with left main coronary disease. Likewise, we selected CAD cases from the smaller GWAS sub-study to serve as the replication dataset $(n=404)$.

\section{Exclusion Criteria}

As we were specifically interested in survival outcomes among people with $\mathrm{CAD}$, we excluded the non-CAD controls from our analysis. We excluded subjects for whom valvular heart disease was either the primary or secondary indication for coronary catheterization and those who had pulmonary hypertension, transplant, right heart catheterization, congenital heart disease, severe congestive heart failure, or peripheral arterial disease intervention. We excluded participants from analyses if they died within 14 days of their initial catheterization in order to mitigate any undue influence of mortality due to procedural intervention on the time-to-event results.

\section{Data Sources, Variables, and Outcomes}

We used de-identified data for our analyses. We determined survival event data as number of days from study enrollment (baseline) to all-cause mortality (event) or last follow-up (censor). Clinical and medical history data came from the Duke Databank for Cardiovascular Disease, the data repository for the primary CATHGEN study.

All patients in CATHGEN had one 6-month follow-up and then annual follow-ups for all-cause mortality, with a maximum of 12 years of follow-up. Study staff adjudicated death events via National Death Index searches, supplementing with Social Security Death Index searches (Sutton et al., 2008).

Covariables for this project were measured for the CATHGEN clinical biorepository, as previously described (Wang et al., 2007). The following were dichotomous variables (yes/no) obtained by medical providers from the participants' detailed medical history: smoking, type 2 diabetes, hyperlipidemia, and hypertension. Clinically defined continuous variables were BMI, creatinine, and ejection fraction.

\section{GWAS Genotyping}

All GWAS genotyping was performed prior to this secondary data analysis project. Sample collection, processing, genotyping and quality control (QC) were performed for the primary CATHGEN study in the Molecular Genomic Core at the Duke Molecular Physiology Institute as previously described (Sutton et al., 2008; Kraus et al., 2015a,b). Post-quality-control genotype data were made available for this project via the Duke PEDIGENE ${ }^{\circledR}$ biorepository database. Genotypes were called using Illumina's GenomeStudio V2010.2 software (version 
1.7.4 Genotyping module). SNPs with $<98 \%$ call frequency, MAF $<0.01$ or that were out of Hardy-Weinberg equilibrium $\left(p<10^{-6}\right)$ were excluded, resulting in 785,945 autosomal SNPs for our GWA screening analysis. Samples with $<98 \%$ call rates for all SNPs, gender mismatches, cryptic relatedness, or outlying ethnicity were excluded (172 samples). We performed secondary analyses of these GWAS data for our discovery and replication cohorts, testing variants having MAF $>0.01$ among White CAD subjects.

\section{Statistical Analyses}

Statistical analyses were performed with the R package using the following stepwise approach (R Core Development Team, 2013). We employed a two-step genome-wide screening, analyzing first the discovery and then the replication datasets for base- and clinical-covariate models. We then performed a combined metaanalysis of the two datasets.

We calculated means and frequencies for baseline demographic variables, diagnoses, and events. We defined time to event as the number of days from study enrollment (time at coronary catheterization and blood collection) to death from any cause. Data from surviving individuals were censored on the date of the last follow-up, consistent with our "survivorship with CAD” phenotype (Dungan et al., 2016). For our analyses of SNPs, we assumed an additive genetic model based on preliminary data demonstrating additive genetic effects (Dungan et al., 2016). We assigned wild-type genotype carriers a value of 0 , heterozygous genotype carriers a value of 1 , and homozygous carriers of the minor allele a value of 2 (Balding, 2006).

In order to determine CAD-specific genetic effects on survival, we employed Cox multivariate regression models to estimate instantaneous risk (hazard) of all-cause mortality among individuals with $\mathrm{CAD}$ by genotype group. For the initial screen, we fit a minimally adjusted (base) model controlling for age, sex, and four principal components of ancestry observed within this White sample. Additional models adjusted for clinical variables controlled for BMI, history of smoking, type 2 diabetes, hyperlipidemia, hypertension, creatinine, and ejection fraction. We used the same analyses in the replication cohort. $P$-values from suggestive discovery and replication results were meta-analyzed via the METAL combined z-score approach (Willer et al., 2010). Only variants that showed the same direction of effect in the discovery and replication phases were included in the meta-analysis phase. Our goal was to identify the strongest candidates with the most consistent findings across the three phases. For top variants, we used Kaplan-Meier curves to show survival probabilities by genotype.

\section{Statistical Screening Thresholds}

Our target association level was the standard GWAS threshold, $p \times 10^{-8}$. Where this stringent threshold was not met, we accepted variants meeting $p \times 10^{-4}$ or less in the discovery phase, indicating suggestive associations for candidate discovery. In the subsequent replication and meta-analysis phases, our goal was to set the association threshold based on Bonferroni correction for the number of variants tested within that phase. The target Bonferroni threshold for the replication phase was $p<5.3 \times 10^{-4}$ (93 variants tested) and for the meta-analysis phase, $p<1.6 \times 10^{-3}$ (all 30 tested variants).

\section{RESULTS}

\section{Demographics}

We present demographic characteristics in Table $\mathbf{1}$. The discovery dataset had more male individuals than the replication dataset, but were similar in all other characteristics. Within the discovery and replication datasets, non-surviving individuals were, on

TABLE 1| Participant characteristics.

\begin{tabular}{|c|c|c|c|c|}
\hline & \multicolumn{2}{|c|}{$\begin{array}{c}\text { Discovery dataset } \\
\text { White CAD cases (684) }\end{array}$} & \multicolumn{2}{|c|}{$\begin{array}{c}\text { Replication dataset } \\
\text { White CAD cases (404) }\end{array}$} \\
\hline & Alive (525) & Dead (159) & Alive (284) & Dead (120) \\
\hline Age $\pm S D$, (range) & $63.32 \pm 10.66$ & $69.59 \pm 10.78$ & $56.99 \pm 9.73$ & $64.43 \pm 11.1$ \\
\hline$C A D$ index $\pm S D$ & $51.32 \pm 17.72$ & $58.29 \pm 20.07$ & $49.48 \pm 17.79$ & $54.88 \pm 19.54$ \\
\hline Male, n (freq) & $388(0.74)$ & $122(0.77)$ & $152(0.54)$ & $59(0.49)$ \\
\hline Smoking, n (freq) & $272(0.52)$ & $85(0.53)$ & $178(0.63)$ & $70(0.58)$ \\
\hline Dyslipidemia, n (freq) & $356(0.68)$ & $108(0.68)$ & $216(0.76)$ & $87(0.73)$ \\
\hline T2DM, n (freq) & $153(0.29)$ & $57(0.36)$ & 89 (0.31) & $54(0.45)$ \\
\hline Hypertension, n (freq) & $357(0.68)$ & $113(0.71)$ & $206(0.73)$ & $91(0.76)$ \\
\hline Ejection fraction $\pm S D$ & $56.55 \pm 12.39$ & $50.37 \pm 15.11$ & $58.05 \pm 10.77$ & $51.51 \pm 14.57$ \\
\hline Creatinine $\pm S D$ & $1.11 \pm 0.66$ & $1.51 \pm 1.23$ & $0.94 \pm 0.21$ & $1.17 \pm 0.76$ \\
\hline
\end{tabular}

CAD, coronary artery disease; SD, standard deviation; BMI, body mass index; T2DM, Type 2 diabetes mellitus; MI, myocardial infarction (ever); ICC, intra-coronary procedures (percutaneous coronary trans/uminal angiography/stent); CABG, coronary artery bypass graft. 
average, older, had more severe CAD (greater CAD index), had a slightly higher prevalence of diabetes and hypertension, and had worse cardiac ejection fraction and renal function (creatinine levels) compared to their survived counterparts.

\section{Follow-Up Events}

In the discovery dataset $(n=684)$, the median follow-up time was 2,004 days (5.5 years) and maximum follow-up was 3,953 days (10.8 years). At the time of analysis, 159 individuals (23.3\%) were deceased on follow-up. In the replication dataset $(n=404)$, the median follow-up was 3,326 days (9.1 years) and the maximum follow-up was 4,420 days (12 years). At the time of analysis, 120 individuals (29.7\%) from the replication cohort were deceased on follow-up.

\section{Genome-Wide Variants Associated With Survival Among Individuals With CAD}

The discovery analysis Q-Q plot indicated that our observed genome-wide signal was consistent with the expected distribution under the null hypothesis (Supplementary Figure 1). The Manhattan plot (Figure 2) shows the negative log p-value for each SNP by chromosome. Given the exploratory aim of this genomewide screen, we have chosen to report the top variants at each phase of the analysis that had consistent directions of effect across the discovery and replication phases.

\section{Discovery}

No SNPs met the standard $10^{-8}$-value threshold (Supplementary Table 1) for the base model analyzed within the discovery dataset; therefore, we moved forward 93 SNPs that had suggestive associations ( $p \times 10^{-4}$ or less) with allcause mortality (Supplementary Table 2). After controlling for clinical covariates, we found that the top three discovery variants (rs7138358, rs7305831, and rs12579455) achieved the accepted genome-wide threshold of $p \times 10^{-8}$ (Table 2). These three variants mapped to the same gene region on chromosome 12q21 (MIR1251/RMST) and were determined to be in linkage disequilibrium, having $r^{2}>0.85$ in European Americans (Machiela and Chanock, 2018).

\section{Replication}

Neither base models nor clinically adjusted models produced SNPs meeting our target Bonferroni threshold for replication of $p<5.3 \times 10^{-4}$. The top two discovery SNPs that had consistent directions of effect in both cohorts met nominal significance $(p<0.05)$ for replication (rs13007553 and rs587936; Table 2). The RMST discovery variants that met the threshold of adjusted $p \times 10^{-8}$ (rs7138358, rs7305831, and rs12579455) did not achieve replication threshold of $p<5.3 \times 10^{-4}$; however, they did meet the meta-analysis threshold of $p<1.6 \times 10^{-3}$.

We observed only nominal significance levels $(p<0.05)$ in the replication, causing concern for the suitability of the chosen replication dataset. In order to evaluate for the congruence of SNP effects between both datasets, we generated an effect size scatterplot (Supplementary Figure 2) for the SNPs having a MAF $>0.05$, adjusted $p<0.05$ and HR $<40$ (1,229 SNPs), as these represented the most stable exploratory results. Specifically, we plotted each SNP's HR for the discovery dataset on the $\mathrm{x}$-axis and their HR for the replication dataset on the $\mathrm{y}$-axis. Supplementary Figure 2 shows multiple SNPs having $p<0.05$ in both datasets with congruent effect sizes between both the discovery and replication datasets (red circles). Supplementary Table 3 contains the detailed results for the 1,229 SNPs from Supplementary Figure 2.

\section{Meta-Analysis}

Of the discovery-identified SNPs, 30, representing 21 distinct gene regions, met the threshold for significance in our metaanalysis $p<1.6 \times 10^{-3}$ and had consistent directions of effect across the two cohorts (Table 2). Only two of these variants (mapped to the same gene) were rare $(\mathrm{MAF}=0.02)$;

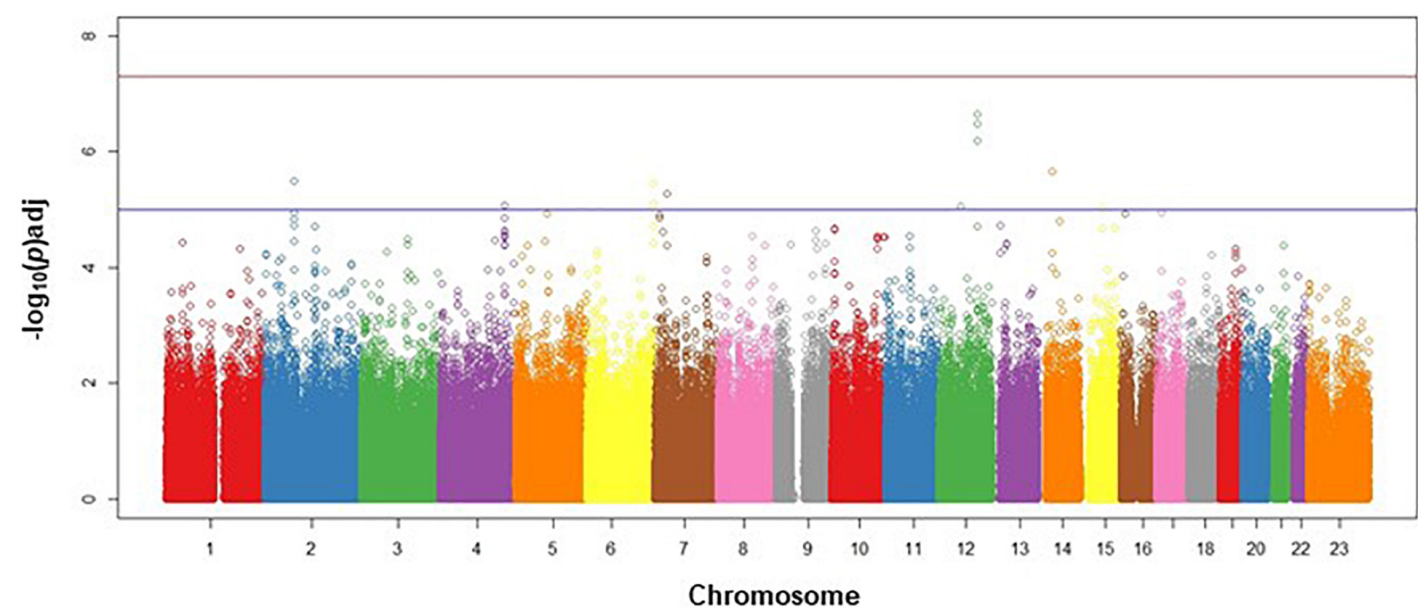

FIGURE 2 | Manhattan plot for discovery phase. Manhattan plot of discovery phase SNPs in genomic order by chromosome and position on the chromosome (X-axis), plotted against the negative log (adj)p-value of each SNP's association with survival events in CAD cases, adjusted for age, sex, and ancestry. Lower horizontal (blue line) represents $p=10^{-5}$; upper horizontal (red) line represents $p=5 \times 10^{-8}$. 
TABLE 2 | Clinically adjusted hazards of all-cause mortality among CAD cases for discovery-screened SNPs.

\begin{tabular}{|c|c|c|c|c|c|c|c|c|c|c|c|}
\hline \multirow[t]{2}{*}{ SNP } & \multirow[t]{2}{*}{ Allelic Variation } & \multirow[t]{2}{*}{ Chr:loc } & \multirow[t]{2}{*}{ Variant } & \multirow[t]{2}{*}{ MAF } & \multirow[t]{2}{*}{ Gene } & \multicolumn{3}{|c|}{$p$-value ${ }^{\dagger}$} & \multirow[t]{2}{*}{ HR } & \multirow[t]{2}{*}{$95 \% \mathrm{Cl}$} & \multirow[t]{2}{*}{$Z$-score } \\
\hline & & & & & & Discovery & Replication & Meta-analysis & & & \\
\hline \multicolumn{12}{|c|}{ Variants with Negative Direction of Effect $(H R<1)$} \\
\hline rs587936 & $\mathrm{T}>\mathbf{A}, \mathbf{C}, \mathbf{G}$ & $9: 121582553$ & Intronic & 0.38 & $D A B 2 I P$ & $4.79 \times 10^{-4}$ & 0.02 & $2.25 \times 10^{-5}$ & 0.65 & $0.51-0.83$ & -4.24 \\
\hline rs118207 & $\mathrm{C}>\mathbf{T}$ & 2:33112067 & Intronic & 0.42 & LTBP1 & $4.43 \times 10^{-4}$ & 0.32 & $7.11 \times 10^{-4}$ & 0.66 & $0.52-0.83$ & -3.39 \\
\hline rs9531515 & $T>\mathbf{A}, \mathbf{G}$ & 13:31380652 & Intergenic & 0.43 & B3GALTL/RXFP2 & $9.43 \times 10^{-6}$ & 0.40 & $5.78 \times 10^{-5}$ & 0.56 & $0.44-0.72$ & -4.02 \\
\hline rs31269 & $G>A, C$ & $5: 76330127$ & Intronic & 0.50 & SV2C & $2.35 \times 10^{-5}$ & 0.41 & $1.17 \times 10^{-4}$ & 0.60 & $0.47-0.76$ & -3.85 \\
\hline rs349443 & $G>T$ & $1: 41811725$ & Intronic & 0.38 & HIVEP3 & $7.19 \times 10^{-5}$ & 0.56 & $4.68 \times 10^{-4}$ & 0.59 & $0.45-0.76$ & -3.50 \\
\hline rs242413 & $C>\mathbf{T}$ & 14:56161083 & Intronic & 0.31 & PELI2 & $7.67 \times 10^{-7}$ & 0.71 & $3.35 \times 10^{-5}$ & 0.49 & $0.36-0.65$ & -4.15 \\
\hline rs7069959 & $\mathbf{T}>\mathrm{C}, \mathrm{G}$ & $10: 130871742$ & Intergenic & 0.27 & GLRX3/MIR378C & $1.85 \times 10^{-4}$ & 0.76 & $1.63 \times 10^{-3}$ & 0.57 & $0.43-0.77$ & -3.15 \\
\hline \multicolumn{12}{|c|}{ Variants With Positive Direction Of Effect (HR > 1) } \\
\hline rs13007553 & $\mathbf{T}>\mathrm{C}$ & $2: 3058976$ & Intronic & 0.36 & LINC01250 & $1.07 \times 10^{-3}$ & 0.03 & $9.53 \times 10^{-5}$ & 1.47 & $1.17-1.86$ & 3.90 \\
\hline rs13022539 & $\mathbf{A}>\mathrm{G}, \mathrm{T}$ & $2: 3058283$ & Intronic & 0.49 & LINC01250 & $9.15 \times 10^{-5}$ & 0.15 & $7.08 \times 10^{-5}$ & 1.59 & $1.26-2.01$ & 3.97 \\
\hline rs2108258 & $A>\mathbf{G}$ & $7: 20752375$ & Intronic & 0.08 & ABCB5 & $1.12 \times 10^{-4}$ & 0.16 & $8.61 \times 10^{-5}$ & 1.89 & $1.37-2.61$ & -3.93 \\
\hline rs17164717 & $A>\mathbf{G}$ & 7:11527743 & Intronic & 0.02 & THSDTA & $1.77 \times 10^{-6}$ & 0.12 & $2.13 \times 10^{-6}$ & 4.33 & $2.37-7.90$ & -4.74 \\
\hline rs10240390 & $A>\mathbf{C}, \mathbf{G}$ & 7:11527648 & Intronic & 0.02 & THSD7A & $2.27 \times 10^{-6}$ & 0.21 & $6.40 \times 10^{-6}$ & 4.28 & $2.34-7.83$ & -4.51 \\
\hline rs1865093 & $C>\mathbf{T}$ & 19:39445494 & $2 \mathrm{~Kb}$ upstream & 0.17 & SUPT5H & $6.33 \times 10^{-4}$ & 0.26 & $6.79 \times 10^{-4}$ & 1.66 & $1.24-2.23$ & 3.40 \\
\hline rs7037490 & $\mathbf{A}>\mathrm{C}, \mathrm{G}$ & 9:38085787 & Intergenic & 0.16 & $S H B / A L D H 1 B 1$ & $1.07 \times 10^{-3}$ & 0.28 & $1.12 \times 10^{-3}$ & 1.59 & $1.20-2.10$ & 3.26 \\
\hline rs7305964 & $\mathbf{G}>\mathrm{A}$ & 12:97499951 & Intronic & 0.32 & $R M S T$ & $1.00 \times 10^{-5}$ & 0.31 & $3.78 \times 10^{-5}$ & 1.71 & $1.35-2.18$ & -4.12 \\
\hline rs17009399 & $G>C$ & 2:73829186 & 5-prime UTR & 0.08 & STAMBP & $9.30 \times 10^{-5}$ & 0.35 & $2.44 \times 10^{-4}$ & 2.01 & $1.42-2.85$ & -3.67 \\
\hline rs744680 & $G>A$ & $10: 129943431$ & Intronic & 0.20 & EBF3 & $2.78 \times 10^{-5}$ & 0.38 & $1.12 \times 10^{-4}$ & 1.74 & $1.34-2.25$ & 3.86 \\
\hline rs12620516 & $G>C, T$ & $2: 216553597$ & Intergenic & 0.29 & RPL37A/IGFBP2 & $7.40 \times 10^{-4}$ & 0.42 & $1.58 \times 10^{-3}$ & 1.50 & $1.19-1.90$ & 3.16 \\
\hline rs1865090 & $T>\mathbf{A}, \mathbf{C}$ & 19:39441383 & Intergenic & 0.17 & RPS16/SUPT5H & $7.40 \times 10^{-4}$ & 0.43 & $1.62 \times 10^{-3}$ & 1.65 & $1.23-2.22$ & -3.15 \\
\hline rs4802033 & $G>A$ & 19:39489326 & Intergenic & 0.17 & TIMM50 & $3.71 \times 10^{-4}$ & 0.46 & $1.06 \times 10^{-3}$ & 1.69 & $1.27-2.26$ & 3.27 \\
\hline rs7305831 & $\mathrm{T}>\mathbf{C}$ & 12:97490908 & Intronic & 0.46 & RMST & $4.30 \times 10^{-8 *}$ & 0.48 & $1.80 \times 10^{-6}$ & 1.98 & $1.55-2.52$ & 4.78 \\
\hline rs7138358 & $\mathbf{C}>\mathrm{T}$ & 12:97491385 & Intronic & 0.46 & RMST & $5.03 \times 10^{-8 *}$ & 0.51 & $2.29 \times 10^{-6}$ & 1.97 & $1.55-2.52$ & -4.73 \\
\hline rs10819587 & $G>A$ & 9:99019019 & Intronic & 0.11 & COL15A1 & $1.82 \times 10^{-4}$ & 0.58 & $9.62 \times 10^{-4}$ & 1.80 & $1.32-2.45$ & 3.30 \\
\hline rs4150403 & $C>\mathbf{T}$ & $2: 127292492$ & Intronic & 0.09 & ERCC3 & $5.62 \times 10^{-5}$ & 0.58 & $4.13 \times 10^{-4}$ & 2.04 & $1.44-2.89$ & 3.53 \\
\hline rs2297603 & $G>\mathbf{A}, \mathbf{T}$ & 9:99015983 & Exonic-missense & 0.11 & COL15A1 & $2.49 \times 10^{-4}$ & 0.59 & $1.22 \times 10^{-3}$ & 1.77 & $1.31-2.41$ & 3.23 \\
\hline rs12579455 & $A>\mathbf{G}, \mathbf{T}$ & 12:97481554 & Intronic & 0.46 & $R M S T$ & $7.927 \times 10^{-8 *}$ & 0.60 & $4.82 \times 10^{-6}$ & 1.95 & $1.53-2.49$ & 4.57 \\
\hline rs12515837 & $T>\mathbf{A}, \mathbf{C}$ & 5:30940209 & Intergenic & 0.05 & LOC729862/CDH6 & $2.75 \times 10^{-4}$ & 0.64 & $1.51 \times 10^{-3}$ & 2.21 & $1.44-3.39$ & -3.17 \\
\hline rs17009433 & $\mathrm{T}>\mathbf{C}$ & 2:73872884 & Intronic & 0.10 & STAMBP & $9.15 \times 10^{-5}$ & 0.70 & $4.13 \times 10^{-3}$ & 1.87 & $1.37-2.55$ & -2.87 \\
\hline rs896651 & $\mathbf{T}>A, G$ & 18: 61564558 & Intergenic & 0.35 & CDH2O/RNF152 & $2.46 \times 10^{-5}$ & 0.70 & $3.46 \times 10^{-4}$ & 1.66 & $1.31-2.10$ & 3.58 \\
\hline rs11126419 & $C>\mathbf{T}$ & $2: 73864992$ & Intronic & 0.09 & STAMBP & $4.29 \times 10^{-5}$ & 0.72 & $5.32 \times 10^{-4}$ & 2.07 & $1.46-2.94$ & 3.46 \\
\hline
\end{tabular}

Less frequent (minor) allele bolded. Bold numerals indicate $p<0.05$.

*indicates GWAS significance threshold $p<10^{-8}$.

${ }^{+}$Results for statistical model controlling for age, sex, and four principal components of ancestry (base model) plus additional clinical covariates: body mass index (BMI), history of smoking, type 2 diabetes, hyperlipidemia, hypertension, creatinine, and ejection fraction. SNP, single nucleotide polymorphism; Chr, chromosome; loc, genomic location (GRCh38.p12); MAF, minor allele frequency; HR, hazard ratio; Cl, confidence interval; UTR, untranslated region; Kb, kilobase. 
all other variants were common in the combined sample with $\mathrm{MAF}>0.05$. Only two variants were significant across all analyses (discovery, replication, and meta-analyses) - one demonstrating decreased and the other increased risk for allcause mortality, adjusted for clinical covariates (Table 2).

The variant significantly associated with increased risk by genotype was rs13007553. Among White CAD cases, each copy of the minor $T$ allele for rs 13007553 conferred 53\% increased risk of all-cause mortality (Figure 3A) in the clinically adjusted model $\left(\right.$ HR 1.47, 95\% CI 1.17-1.86, $p($ adj $)=1.07 \times 10^{-3}$ [discovery], $p($ adj $)=0.03$ [replication], $p($ adj $)=9.53 \times 10^{-5}$ [metaanalysis]). The rs13007553 SNP is located in LINC01250, or long intergenic non-protein coding RNA 1250. This common intronic SNP $(\mathrm{MAF}=0.36)$ on Chromosome 2p25.3 (GRCh38.p12 build position 3058976) resides between MYT1L and EIPR1 (alias TSSC1). Another intergenic variant for LINC01250 (MYT1L/EIPR1; rs13022539) was significant in the discovery dataset $\left(p=6.03 \times 10^{-5} ; p(\right.$ adj $\left.)=9.15 \times 10^{-5}\right)$ and metaanalysis $\left(p=7.08 \times 10^{-5}\right)$ but did not reach significance in the replication dataset $(p=0.15)$. However, these two SNPs are in linkage disequilibrium among European samples $\left(r^{2}=0.53\right.$, $D^{\prime}=0.96, p<0.0001$ ) indicating that they may represent the same underlying genetic effect (Machiela and Chanock, 2018). There is no functional annotation for rs13007553, and it has no

\section{A} rs13007553 (MYT1L/EIPR1)
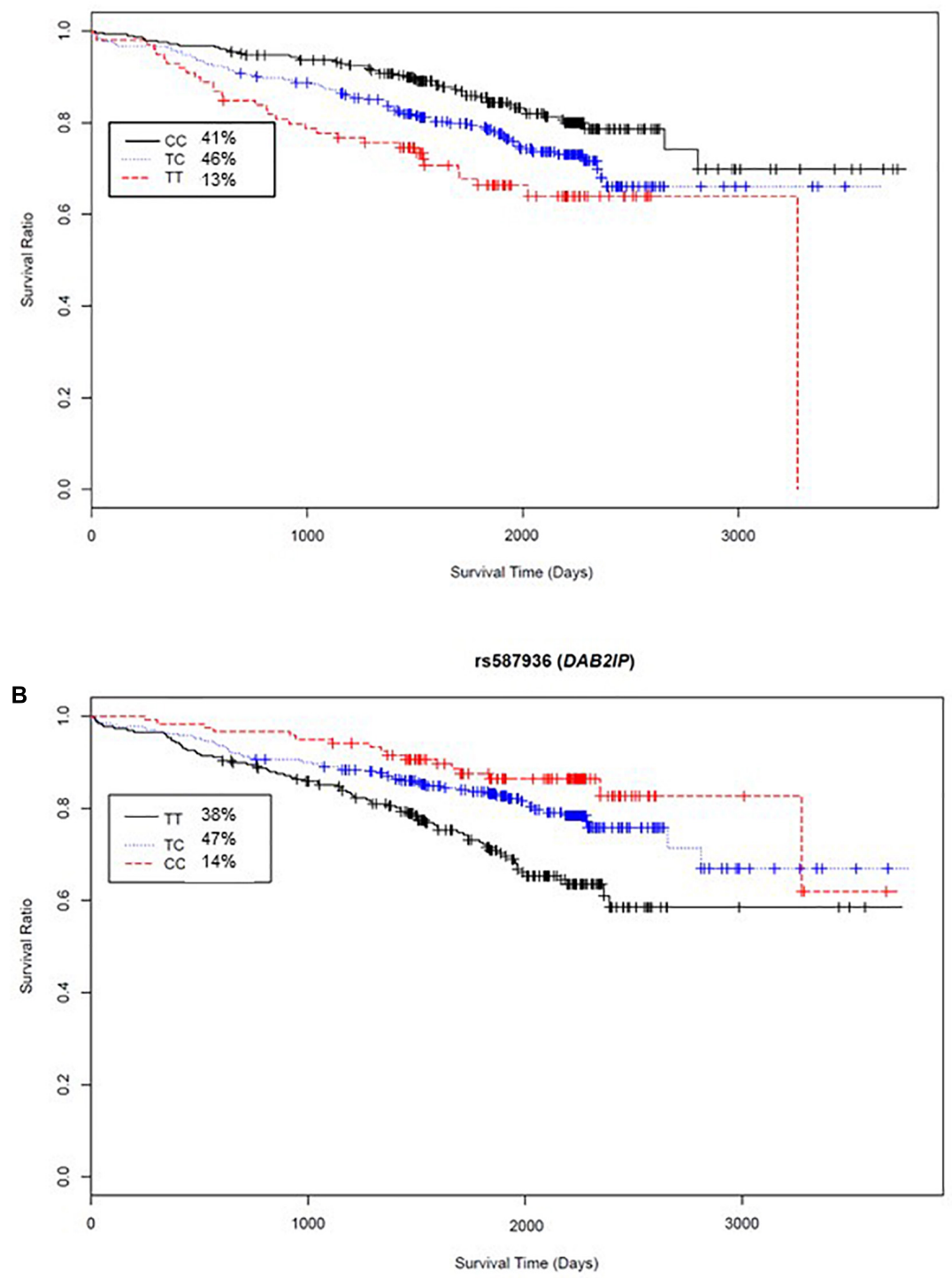

FIGURE 3 | Kaplan-Meier curves for top two meta-analyses genome-wide SNP exemplars. $X$-axis, days from enrollment to death or last follow up; $Y$-axis, probability of survival; inset box, genotype frequencies. (A) Survival curve by rs 13007553 genotype indicates 1.47 times increased hazard of all-cause mortality for each addition of the minor allele (T nucleotide). (B) Survival curve by rs587936 genotype indicates reduced hazard of all-cause mortality with each addition of the minor allele (C nucleotide) (HR 0.65). 
expression quantitative trail loci (eQTL) relationships according to the Genotype-Tissue Expression project (GTEx).

Conversely, rs587936 demonstrated attenuated risk by number of minor alleles. In our analysis, each copy of the minor C allele for rs587936 conferred significantly reduced risk of all-cause mortality (Figure 3B; HR 0.65, 95\% CI0.51-0.83, $p($ adj $)=4.79 \times 10^{-4}$ [discovery], $p($ adj $)=0.02$ [replication], $p($ adj $)=2.25 \times 10^{-5}$ [meta-analysis] $)$, controlling for clinical covariates. The rs587936 marker is a common intronic SNP $(\mathrm{MAF}=0.38)$ on Chromosome 9q33.2 (GRCh38.p12 build position 121582553) mapped to the first intron of DAB2IP (alias AIP1). There is no functional annotation for rs587936 and no evidence of expression quantitative trait loci (eQTL) with DAB2IP (GTEx database). However, further review of gene expression data in the GTEx database shows modest evidence that rs587936 is associated with expression of the pseudogene GGTAP1 in heart tissue and expression of an uncharacterized locus, LOC102723324, in multiple tissues including adipose, lung, and aorta.

\section{DISCUSSION}

In the present study, we conducted genome-wide discovery, replication, and meta-analysis screening for genetic contribution to differential survival outcomes among 1,088 White patients with clinically significant CAD from the southeastern United States. We observed improved $p$-values after controlling for the clinical covariates but only minimal shifts in the effect sizes. For this reason, we discuss the clinically adjusted models here and provide the base-adjusted results as Supplementary Material. Our major findings are the identification of two common gene variants consistently associated with risk for all-cause mortality among White patients with CAD. The minor C allele for DAB2IP (AIP1) rs587936 was associated with significantly reduced risk of all-cause mortality; whereas, the minor $\mathrm{T}$ allele for $\mathrm{rs} 13007553$, residing between the MYTIL and EIPR1 genes, conferred significantly increased risk for all-cause mortality.

While our findings offer substantive evidence of an association between rs587936 ( $\mathrm{HR}<1)$ and survival outcomes among White individuals with CAD, van der Harst and Verweij (2018) reported that DAB2IP rs885150 was associated with reduced risk of $\mathrm{CAD}(\mathrm{OR}<1)$ in a recent GWAS of UK Biobank and CARDIoGRAMplusC4D (OR0.96, 95\% CI0.95$\left.0.98, p=2.1 \times 10^{-8}\right)$. In prior studies, researchers had reported $D A B 2 I P$ association with lower-limb ischemia and CAD case status (Harrison et al., 2012; Smith and Newton-Cheh, 2015), and with aortic aneurysm (Gretarsdottir et al., 2010), all of which increase mortality risk in patients with CAD. The most commonly studied variant of DAB2IP, rs7025486, is associated with cardiac risk phenotypes and is part of a 21gene risk score that significantly predicts CAD case status. It was significantly associated with blood lipid levels in a cohort of Pakistani people, but was not an independent predictor as a single SNP (Shahid et al., 2017). We determined that rs7025486 and rs587936 are not in linkage disequilibrium $\left(r^{2}=0.0001\right.$, $\left.d^{\prime}=0.0145, p=0.547\right)$, suggesting allelic heterogeneity in this region (Machiela and Chanock, 2018).

$D A B 2 I P$ is a ras/GAP tumor suppressor gene that encodes an ASK1-interacting protein abundantly expressed in vascular endothelial cells (Zhang et al., 2015). DAB2IP is an alias for $A S K-1$, which is broadly termed AIP1 (anti-inflammatory protein-1) for its so-named properties (Zhang et al., 2015). In vitro and murine knockout studies support AIP1 as "a signaling adaptor molecule implicated in stress and apoptotic signaling induced by proinflammatory mediators" (Huang et al., 2013, p. 795). DAB2IP-encoded ASK1/AIP1 also suppresses atherosclerosis by limiting hyperlipidemia-induced inflammation and vascular endothelial dysfunction (Huang et al., 2013). These reported relationships between DAB2IP and vascular anti-inflammatory processes offer biologic plausibility for gene variation in DAB2IP conferring increased survival time for people with $\mathrm{CAD}$. Additional replication and validation are required to test hypotheses about the functional relation of $D A B 2 I P$ on survival.

Consistent with its earlier recognition as a ras/GAP tumor suppressor, $D A B 2 I P$ also is associated with risk for various cancers and cancer cell proliferation and migration (Zhou et al., 2015; Li et al., 2016; Olsen et al., 2017). Among its effects, DAB2IP inhibits oncogenic processes and facilitates cancer cell apoptosis (Liu et al., 2016). In our earlier study in which we used a candidate gene approach, we found the tumor suppressor gene LSAMP to be a statistically significant marker of survivorship with CAD (Dungan et al., 2013). Our present identification of an association between another tumor-suppressor gene, DAB2IP, and CAD survival outcomes in the present GWA screening underscores the importance of considering antagonistic pleiotropy when seeking to identify genetic effects in complex diseases. Antagonistic pleiotropy refers to a gene having dual effects such that it may confer both enhanced fitness via effects on a beneficial trait and increased risk for a detrimental trait. Murine knockout studies provided early evidence that DAB2IP genetic effects may be pleiotropic. Both global gene knockouts and deletion of $D A B 2 I P$ specifically in vascular endothelial cells consistently resulted in enhanced inflammatory responses, atherosclerosis exacerbation, and graft arteriosclerosis progression (Zhang et al., 2015). However, DAB2IP loss also conferred resistance to prostate cancer through modulation of apoptosis (Zhou et al., 2015). Theoretically, a mouse having no ability to produce DAB2IP might simultaneously develop hallmark signs of CAD (inflammation, athero- and arteriosclerosis) and protection against the development of prostate cancer. It is unclear whether antagonistic pleiotropy in animal models extrapolates to humans. However, other examples of antagonistic genetic effects involving CAD and cancer risk exist in human cohort studies (Kulminski et al., 2011). Further work is needed to expand our understanding of the complex relationships among DAB21P (AIP1), CAD, cancer, and survival.

The other variant consistently associated with survival in CAD in the present study, the intergenic rs13007553 (LINC01250) located between MYT1L and EIPR1 (alias TSSC1), was linked with increased risk of all-cause mortality among CAD cases. The biologic relevance is unclear regarding these genes nearby 
to MYT1L and our CAD survival phenotype. Similar to the candidate gene LSAMP identified in our prior studies of survival with CAD (Dungan et al., 2013, 2016), MYT1L plays a key role in neuronal differentiation. Variation in MYT1L is implicated in fibromyalgia and is associated with a nonspecific clinical phenotype that includes intellectual disability, early-onset obesity, and speech delay (Docampo et al., 2014; Al Tuwaijri and Alfadhel, 2019). EIPR1 (alias TSSC1, the other gene flanking rs13007553), is involved in endosomal retrieval pathways (Gershlick et al., 2016). In vitro experiments have demonstrated that TSSC1 can regulate metastatic properties in breast cells (Wang et al., 2013). Our findings in the present study add evidence to the literature regarding the association of rs13007553 (i.e., the MYT1L/EIPR1 intergenic region) with CAD-related survival outcomes.

Additional genes of interest in our results are rhabdomyosarcoma 2 associated transcript (RMST) and thrombospondin type-1 domain-containing protein 7A (THSD7A). Specifically, four RMST SNPs (rs7305964, rs7305831, rs7138358, and rs12579455) were associated with increased risk of all-cause mortality in the White CAD patients we analyzed (Table 2). The latter three SNPs were our top discovery hits. Models adjusted for clinical covariates for these three variants resulted in $p$-values meeting the stringent $10^{-8}$ threshold for significance. In a previous study, researchers reported a significant association between another GWAS-identified RMST SNP, rs10777845, and increased risk of sudden cardiac arrest among patients with CAD (OR 1.12, 95\% CI 1.071.17; $p=5.0 \times 10^{-6}$; Li et al., 2018). The mechanisms for RMST contribution to cardiovascular diseases are unknown. Another two of the SNPs with a significant association to CAD survival in the discovery phase of the present study annotate to THSD7A (rs17164717, rs10240390). THSD7A variation was associated with CAD in a GWAS of over 21,000 Han Chinese participants (rs17165136; OR 1.28, 95\% CI 1.21-1.35; $p<1.00 \times 10^{-25}$; Aouizerat et al., 2011). Subsequent functional studies with knockdown of THSD7A have demonstrated reduced monocyte adhesion via decreased expression of intracellular adhesion molecule-1 (ICAM-1), L-selectin, and integrin subunit beta 2 (ITGB2) (Li et al., 2018). The present study offers supportive evidence for THSD7A and RMST candidacy in cardiovascular disease.

Given the potential for shared genetic variation among phenotypes for survivorship with CAD and longevity, we also assessed whether our top hits with increased survival time $(H R<1)$ had previous associations with longevity phenotypes. We found no such associations in the NHGRI-EBI Catalog of human GWASs. Nor were the loci of these variants near the mostvalidated longevity candidate regions (Pilling et al., 2017; Deelen et al., 2019; Zenin et al., 2019).

\section{STRENGTHS AND LIMITATIONS}

Results of the present exploratory genome-wide screen add new insights to the limited literature on the associations of CAD candidate genes with survival outcomes, most notably regarding improved survival outcomes. We found only one other phenotypically similar candidate study in the literature. In that study, researchers reported that SDF1 (alias, CXCL12) conferred significantly improved event-free survival among patients with symptomatic $\mathrm{CAD}$, even after controlling for clinical covariates (Rath et al., 2016).

Our results also add to the literature on the genetic candidates for increased risk for mortality in CAD. The most rigorous candidate evidence to date comes from a resequencing of APOA1 in which investigators identified a novel mutation that predicted a 2.5 -fold increased hazard of death with a mean reduction of 10 years in survival time in heterozygous carriers among participants in the Copenhagen City Heart Study, who were followed for 31 years (Haase et al., 2011).

Our use of an extensive clinical cardiovascular biorepository having genome-wide participant data and a maximum of 12 years of longitudinal follow-up was both a strength and a limitation of the present study. While this resource allowed us to explore genomic variation associated with longitudinal survival outcomes among people with prevalent CAD, the medical history variables were not defined and measured from a research perspective, limiting our ability to explore the effects of these factors. For example, smoking history was operationalized as a self-reported "yes/no" variable obtained by provider intake assessment rather than being measured for optimal internal validity using additional variables such as ever/current, frequency, and exposure type or interval (Florescu et al., 2009). Additionally, dietary variables were not measured in CATHGEN. Given the importance of smoking and diet as risk factors for CAD (McGillicuddy and Roche, 2012; Gambardella et al., 2017) and as having potential gene-interaction effects (Horne et al., 2009), these gaps in the database are a limitation for the present study.

Because our goal in the present study was to add to the literature with an exploratory GWA screen for the CAD survival phenotype, we decided to relax the $p$-value thresholds and focus on the top-most variants with consistent directions of effect across the analysis phases. We readily acknowledge that our decision to relax the $p$-value thresholds for the discovery and replication phases represents a limitation. We offer two points of consideration: First, our concern about this limitation prompted us to evaluate the 1,229 most stable SNPs (MAF $>0.05$, adjusted $p$-values $<0.05$ and HR $<40$ ) for the presence and quality of effect size congruence between both of the datasets (Supplementary Figure 2). The scatterplot demonstrates the extent to which the SNP effects correlate between the discovery and replication analyses. We are encouraged by the numerous SNPs having similar effect sizes in the replication dataset as they had in the discovery dataset (red circles). Second, Purcell et al. (2009) set an important precedent for relaxing discovery $p$-values when SNPs for schizophrenia identified in the discovery phase of their GWAS did not meet the stringent $10^{-8}$ GWAS $p$-value threshold, yet were later replicated and also demonstrated significance as part of a genomic risk score for schizophrenia and other related phenotypes (Marees et al., 2018). Future studies 
designed specifically for the aim of identifying genome-wide association with survival outcomes among patients with CAD should strive for the more stringent $10^{-8} p$-value criterion typically expected for GWAS-level significance. Our results in the present study identify candidate genes for further investigation. To support such work, we offer details of variant annotations, effect sizes, and $p$-values in Supplementary Table 3. We continue to seek opportunities for additional meta-analyses and have constructed our results tables to facilitate such analyses.

Differences in clinical characteristics and study design between the discovery and replication cohorts are likely culprits in the limited number of replicated variants observed. The potential exists for confounding between SNP variation and untested clinical covariates and/or survival outcomes. Of note, CATHGEN CAD cases are defined by the Duke CAD index, a measure of CAD severity which accounts for both the extent and location of coronary stenosis. Since disease severity was used to determine case status in the original sub-studies, it would not have been prudent to use disease severity as a covariant in the models in the present study. Therefore, we could not evaluate the specific influence of disease severity on survival outcomes in the present sample. Similarly, we could not evaluate the specific influence of left main arterial stenosis on survival within the present study due to the limited number of individuals with this severe phenotype. Addressing this limitation in future research would improve upon our understanding of disease severity and stenosis location as mediators or moderators of genetic effects on survival outcomes. Additionally, our findings may represent genetic signal for unstudied factors that may co-occur among non-surviving patients with CAD.

Treatment effects may have confounded the survival rates in the CATHGEN clinical observational studies from which we drew the data for the present analysis. We were unable to control for medical history of myocardial infarction and interventional treatment via $C A B G$ or stent placement due to excessive missing data; attempting to include these variables in our statistical models resulted in model divergence. We were also unable to control for the use of medications such as aspirin and other antiplatelet agents, statins, and beta-blockers, which are well-established independent predictors of survival and mortality among CAD patients (Antithrombotic Trialists' Collaboration, 2002; Dézsi and Szentes, 2017; Rodriguez et al., 2019). The observed association of rs587936 with improved survival in CAD is likely the most susceptible to confounding by medication effects of the findings in the present study. However, our analyses identified an association between rs13007553 and increased risk of mortality in CAD even with the presumed presence of medication treatment effects. We would expect that controlling for medication use would strengthen the genetic signal of that variant further.

The data source for the present secondary analysis did not include sufficient data to accurately adjudicate cardiac causes of death. As we discussed in a previous study, the most internally valid phenotype definition for survivorship with CAD is the time to death due to CAD-specific causes. In the absence of sufficient data on cause of death, we selected all-cause mortality as a suitable primary endpoint (Dungan et al., 2013). We also must consider the potential for survival bias in genetic-associated endpoints, as we have examined previously (Dungan et al., 2013). Simulated evidence of lethal cardiac events suggests that we can expect an erosion of no more than $20 \%$ in effect size due to survival bias since our cohort's mean age is less than 75 years (Anderson et al., 2011). A more recent study concluded that survival bias was unlikely when genetic effects were small (OR, HR < 2) when examining recurrent events after non-fatal MI (Hu et al., 2017). In the present study, however, we did not study a conditional, recurrent endpoint.

Our results are only generalizable to White people with CAD in the southeastern United States. We readily acknowledge the need for expanding this work to include ancestrally diverse and marginalized individuals, as they are often at greater risk for both CAD and mortality. Expanding diverse representation for genomic studies is a priority for our future work, and we continue to explore solutions to address sampling and inclusion limitations.

\section{CONCLUSION}

Our goal with the present study was to generate discovery variants as a logical next step to support future hypothesisdriven work. Using genome-wide screening, we identified two candidate gene markers associated with survival outcomes across a 12-year follow-up among 1,088 White participants whose CAD was clinically defined via cardiac catheterization. Allelic variation in rs587936 (DAB2IP) conferred reduced risk and rs13007553 conferred increased risk for all-cause mortality, even after controlling for clinical covariates. These findings extend prior findings of associations of DAB2IP with CAD phenotypes to include survival in those with CAD. Our observed association between rs13007553 (LINC01250, intergenic of MYT1L/TSSC1) and increased risk of allcause mortality in patients with CAD is a novel finding among the current literature. These candidate variants do not appear to overlap with the top longevity candidate genes. Additional research is needed to identify genetic contributions to survivorship in those with significant CAD as well as the underlying biological mechanisms of those contributions. Our results will serve as a resource for in silico and meta-analyses and can inform the design of future studies. Such work could lead to a better understanding of mortality risk and protective mechanisms in the context of the coronary disease state.

\section{DATA AVAILABILITY STATEMENT}

The original contributions presented in the study are publicly available. These data can be found here: https:// www.ncbi.nlm.nih.gov/projects/gap/cgi-bin/study.cgi?study_id= phs000703.v1.p1 [Accession number phs000703.v1.p1]. 


\section{ETHICS STATEMENT}

The studies involving human participants were reviewed and approved by Duke University Medical Center Institutional Review Board. The patients/participants provided their written informed consent to participate in this study.

\section{AUTHOR CONTRIBUTIONS}

JD, XQ, EH, and WK contributed to conception and design of the study. $\mathrm{CH}$ (senior analyst) procured the database. $\mathrm{MH}$ adjudicated and managed data in preparation for analyses. XQ performed the statistical analysis. JD wrote the first draft of the manuscript. EH and WK contributed substantive edits for the manuscript. All authors contributed to manuscript revision, read, and approved the submitted version.

\section{FUNDING}

Support for this work was provided by the NIH/National Institute of Aging (5T32 AG000029-31; HJ Cohen, PI; JR Dungan, trainee); National Institute of Nursing Research (K99 NR011054/R00NR011054; JR Dungan, PI), Duke University School of Nursing (Pilot Research Grant, JR Dungan, PI), Duke University John A. Hartford Center for Excellence (Junior Faculty Fellowship \#2006-0109; JR Dungan, PI), National Heart, Lung and Blood Institute (R01 HL095987; SH Shah, PI), and (5RC2HL101621-02; WE Kraus, PI). The Genotype-Tissue Expression (GTEx) Project was supported by the Common Fund of the Office of the Director of the National Institutes of Health and by NCI, NHGRI, NHLBI, NIDA, NIMH, and NINDS. The data used for the comparisons described in this

\section{REFERENCES}

Al Tuwaijri, A., and Alfadhel, M. (2019). Myt1L mutation in a patient causes intellectual disability and early onset of obesity: a case report and review of the literature. J. Pediatr. Endocrinol. Metab. 32, 409-413. doi: 10.1515/jpem-20180505

Anderson, C. D., Nalls, M. A., Biffi, A., Rost, N. S., Greenberg, S. M., Singleton, A. B., et al. (2011). The effect of survival bias on case-control genetic association studies of highly lethal diseases. Circ. Cardiovasc. Genet. 4, 188-196. doi: 10.1161/CIRCGENETICS.110.957928

Aouizerat, B. E., Vittinghoff, E., Musone, S. L., Pawlikowska, L., Kwok, P.Y., Olgin, J. E., et al. (2011). GWAS for discovery and replication of genetic loci associated with sudden cardiac arrest in patients with coronary artery disease. BMC Cardiovasc. Disord. 11:29. doi: 10.1186/1471-226111-29

Balding, D. J. (2006). A tutorial on statistical methods for population association studies. Nat. Rev. Genet. 7, 781-791. doi: 10.1038/ nrg1916

Antithrombotic Trialists' Collaboration (2002). Collaborative meta-analysis of randomised trials of antiplatelet therapy for prevention of death, myocardial infarction, and stroke in high risk patients. BMJ 324, 71-86. doi: 10.1136/bmj. 324.7329 .71

Deelen, J., Evans, D. S., Arking, D. E., Tesi, N., Nygaard, M., Liu, X., et al. (2019). A meta-analysis of genome-wide association studies identifies multiple longevity genes. Nat. Commun. 10:3669. doi: 10.1038/s41467-01911558-2 manuscript were obtained from https://gtexportal.org/home/, the GTEx Portal on 01/13/2021.

\section{ACKNOWLEDGMENTS}

The authors wish to express gratitude to the participants of the Duke Catheterization Genetics Study (CATHGEN), without whom this work could not have been possible.

\section{SUPPLEMENTARY MATERIAL}

The Supplementary Material for this article can be found online at: https://www.frontiersin.org/articles/10.3389/fgene. 2021.661497/full\#supplementary-material

Supplementary Figure 1 | Discovery phase Q-Q plot.

Supplementary Figure 2 | SNP effect size congruence between discovery and replication datasets.

Supplementary Table 1 | Base model hazard of all-cause mortality among cad cases for discovery-screened SNPs. Less frequent (minor) allele bolded. Bold numerals indicate $p<0.05$. *Results for base statistical model controlling for age, sex, and four principal components of ancestry. SNP, single nucleotide polymorphism; Chr, chromosome; loc, genomic location (Grch38.p12); MAF, minor allele frequency; HR, hazard ratio; Cl, confidence interval; UTR, untranslated region; Kb, kilobase.

Supplementary Table 2 | Top 93 discovery SNPs. *Base model adjusting for age, sex, and four principal components of ancestry; $p$ (adj), clinically adjusted model controlling for body mass index (BMI), history of smoking, type 2 diabetes, hyperlipidemia, hypertension, creatinine, and ejection fraction. Bold indicates GWAS-level significance $p=10^{-8}$.

Supplementary Table 3 | Details of 1229 SNPs evaluated for effect size congruence.

Dézsi, C. A., and Szentes, V. (2017). The real role of $\beta$-blockers in daily cardiovascular therapy. Am. J. Cardiovasc. Drugs 17, 361-373. doi: 10.1007/ s40256-017-0221-8

Docampo, E., Escaramís, G., Gratacòs, M., Villatoro, S., Puig, A., Kogevinas, M., et al. (2014). Genome-wide analysis of single nucleotide polymorphisms and copy number variants in fibromyalgia suggest a role for the central nervous system. Pain 155, 1102-1109. doi: 10.1016/j.pain.2014.02.016

Dungan, J. R., Qin, X., Hauser, E. R., and Kraus, W. E. (2013). The genetic basis for survivorship in coronary artery disease. Front. Genet. 4:191. doi: 10.3389/fgene. 2013.00191

Dungan, J. R., Qin, X., Horne, B. D., Carlquist, J. F., Singh, A., Hurdle, M., et al. (2016). Case-only survival analysis reveals unique effects of genotype, sex, and coronary disease severity on survivorship. PLoS One 11:e0154856. doi: 10.1371/journal.pone.0154856

Ellis, K., Palmer, B., Frampton, C., Troughton, R., Doughty, R., Whalley, G., et al. (2013). Genetic variation in the renin-angiotensin-aldosterone system is associated with cardiovascular risk factors and early mortality in established coronary heart disease. J. Hum. Hypertens. 27, 237-244. doi: 10.1038/jhh. 2012.24

Figarska, S. M., Vonk, J. M., Van Diemen, C. C., Postma, D. S., and Boezen, H. M. (2013). ADAM33 gene polymorphisms and mortality. A prospective cohort study. PLoS One 8:e67768. doi: 10.1371/journal.pone.0067768

Florescu, A., Ferrence, R., Einarson, T., Selby, P., Soldin, O., and Koren, G. (2009). Methods for quantification of exposure to cigarette smoking and environmental tobacco smoke: focus on developmental toxicology. Ther. Drug Monit. 31:14. doi: 10.1097/FTD.0b013e3181957a3b 
Gambardella, J., Sardu, C., Sacra, C., Del Giudice, C., and Santulli, G. (2017). Quit smoking to outsmart atherogenesis: molecular mechanisms underlying clinical evidence. Atherosclerosis 257, 242-245. doi: 10.1016/j.atherosclerosis.2016.12. 010

Gershlick, D. C., Schindler, C., Chen, Y., and Bonifacino, J. S. (2016). TSSC1 is novel component of the endosomal retrieval machinery. Mol. Biol. Cell 27, 2867-2878. doi: 10.1091/mbc.e16-04-0209

Gioli-Pereira, L., Santos, P. C. J. L., Ferreira, N. E., Hueb, W. A., Krieger, J. E., and Pereira, A. C. (2012). Higher incidence of death in multi-vessel coronary artery disease patients associated with polymorphisms in chromosome 9p21. BMC Dardiovasc. Disord. 12:61. doi: 10.1186/1471-2261-12-61

Gretarsdottir, S., Baas, A. F., Thorleifsson, G., Holm, H., Den Heijer, M., De Vries, J.-P. P., et al. (2010). Genome-wide association study identifies a sequence variant within the DAB2IP gene conferring susceptibility to abdominal aortic aneurysm. Nat. Genet. 42, 692-697. doi: 10.1038/ng.622

Haase, C. L., Frikke-Schmidt, R., Nordestgaard, B. G., Kateifides, A. K., Kardassis, D., Nielsen, L. B., et al. (2011). Mutation in APOA1 predicts increased risk of ischaemic heart disease and total mortality without low HDL cholesterol levels. J. Intern. Med. 270, 136-146. doi: 10.1111/j.1365-2796.2011.02381.x

Harrison, S. C., Cooper, J. A., Li, K., Talmud, P. J., Sofat, R., Stephens, J. W., et al. (2012). Association of a sequence variant in DAB2IP with coronary heart disease. Eur. Heart J. 33, 881-888. doi: 10.1093/eurheartj/ehr075

Horne, B. D., Hauser, E. R., Wang, L., Muhlestein, J. B., Anderson, J. L., Carlquist, J. F., et al. (2009). Validation study of genetic associations with coronary artery disease on chromosome 3q13-21 and potential effect modification by smoking. Ann. Hum. Genet. 73, 551-558. doi: 10.1111/j.1469-1809.2009.00540.x

Hu, Y. J., Schmidt, A. F., Dudbridge, F., Holmes, M. V., Brophy, J. M., Tragante, V., et al. (2017). Impact of selection bias on estimation of subsequent event risk. Circ. Cardiovasc. Genet. 10:e001616. doi: 10.1161/CIRCGENETICS.116.001616

Huang, Q., Qin, L., Dai, S., Zhang, H., Pasula, S., Zhou, H., et al. (2013). AIP1 suppresses atherosclerosis by limiting hyperlipidemia-induced inflammation and vascular endothelial dysfunction. Arterioscle. Thromb. Vasc. Biol. 33, 795804. doi: 10.1161/ATVBAHA.113.301220

Ivanova, A. A., Maksimov, V. N., Orlov, P. S., Ivanoshchuk, D. E., Savchenko, S. V., and Voevoda, M. I. (2017). Association of the genetic markers for myocardial infarction with sudden cardiac death. Indian Heart J. 69, S8-S11. doi: 10.1016/ j.ihj.2016.07.016

Kraus, W. E., Granger, C. B., Sketch, M. H., Donahue, M. P., Ginsburg, G. S., Hauser, E. R., et al. (2015a). A guide for a cardiovascular genomics biorepository: the CATHGEN experience. J. Cardiovasc. Transl. Res. 8, 449-457. doi: 10.1007/s12265-015-9648-y

Kraus, W. E., Muoio, D. M., Stevens, R., Craig, D., Bain, J. R., Grass, E., et al. (2015b). Metabolomic quantitative trait loci (mQTL) mapping implicates the ubiquitin proteasome system in cardiovascular disease pathogenesis. PLoS Genet. 11:e1005553. doi: 10.1371/journal.pgen.1005553

Kulminski, A. M., Culminskaya, I., Ukraintseva, S. V., Arbeev, K. G., Arbeeva, L., Wu, D., et al. (2011). Trade-off in the effects of the apolipoprotein E polymorphism on the ages at onset of CVD and cancer influences human lifespan. Aging Cell 10, 533-541. doi: 10.1111/j.1474-9726.2011.00689.x

Li, X., Dai, X., Wan, L., Inuzuka, H., Sun, L., and North, B. J. (2016). Smurf1 regulation of DAB2IP controls cell proliferation and migration. Oncotarget 7:26057. doi: 10.18632/oncotarget.8424

Li, Y., Wang, D. W., Chen, Y., Chen, C., Guo, J., Zhang, S., et al. (2018). Genomewide association and functional studies identify SCML4 and THSD7A as novel susceptibility genes for coronary artery disease. Arterioscler. Thromb. Vascu. Biol. 38, 964-975. doi: 10.1161/ATVBAHA.117.310594

Liu, L., Xu, C., Hsieh, J. T., Gong, J., and Xie, D. (2016). DAB2IP in cancer. Oncotarget 7, 3766-3776. doi: 10.18632/oncotarget.6501

Machiela, M. J., and Chanock, S. J. (2018). LDassoc: an online tool for interactively exploring genome-wide association study results and prioritizing variants for functional investigation. Bioinformatics 34, 887-889. doi: 10.1093/ bioinformatics/btx561

Marees, A. T., De Kluiver, H., Stringer, S., Vorspan, F., Curis, E., Marie-Claire, C., et al. (2018). A tutorial on conducting genome-wide association studies: quality control and statistical analysis. Int. J. Methods Psychiatr. Res. 27:e1608. doi: $10.1002 / \mathrm{mpr} .1608$
McGillicuddy, F. C., and Roche, H. M. (2012). Nutritional status, genetic susceptibility, and insulin resistance-important precedents to atherosclerosis. Mol. Nut. Food Res. 56, 1173-1184. doi: 10.1002/mnfr. 201100785

Olsen, S. N., Wronski, A., Castaño, Z., Dake, B., Malone, C., De Raedt, T., et al. (2017). Loss of RasGAP tumor suppressors underlies the aggressive nature of luminal B breast cancers. Cancer Discov. 7, 202-217. doi: 10.1158/2159-8290. CD-16-0520

Patel, R. S., Schmidt, A. F., Tragante, V., Mccubrey, R. O., Holmes, M. V., Howe, L. J., et al. (2019). Association of Chromosome 9p21 with subsequent coronary heart disease events: a GENIUS-CHD study of individual participant data. Circ. Genom. Precis. Med. 12:e002471. doi: 10.1161/CIRCGEN.119.002471

Pilling, L. C., Kuo, C.-L., Sicinski, K., Tamosauskaite, J., Kuchel, G. A., Harries, L. W., et al. (2017). Human longevity: 25 genetic loci associated in 389,166 UK biobank participants. Aging 9, 2504-2520. doi: 10.18632/aging.101334

Purcell, S. M., Wray, N. R., Stone, J. L., Visscher, P. M., O’donovan, M. C., Sullivan, P. F., et al. (2009). Common polygenic variation contributes to risk of schizophrenia and bipolar disorder. Nature 460, 748-752. doi: 10.1038/ nature08185

R Core Development Team (2013). R: A Language and Environment for Statistical Computing. Vienna: R Foundation for Statistical Computing.

Rath, D., Schaeffeler, E., Winter, S., Hewer, J., Müller, K., Droppa, M., et al. (2016). SDF1 polymorphisms influence outcome in patients with symptomatic cardiovascular disease. PLoS One 11:e0161933. doi: 10.1371/journal.pone. 0161933

Rodriguez, F., Maron, D. J., Knowles, J. W., Virani, S. S., Lin, S., and Heidenreich, P. A. (2019). Association of statin adherence with mortality in patients with atherosclerotic cardiovascular disease. JAMA Cardiol. 4, 206-213. doi: 10.1001/ jamacardio.2018.4936

Shahid, S. U., Cooper, J. A., Beaney, K. E., Li, K., Rehman, A., and Humphries, S. E. (2017). Genetic risk analysis of coronary artery disease in Pakistani subjects using a genetic risk score of 21 variants. Atherosclerosis 258, 1-7. doi: 10.1016/ j.atherosclerosis.2017.01.024

Smith, J. G., and Newton-Cheh, C. (2015). Genome-wide association studies of late-onset cardiovascular disease. J. Mol. Cell. Cardiol. 83, 131-141. doi: 10. 1016/j.yjmcc.2015.04.004

Sutton, B. S., Crosslin, D. R., Shah, S. H., Nelson, S. C., Bassil, A., Hale, A. B., et al. (2008). Comprehensive genetic analysis of the platelet activating factor acetylhydrolase (PLA2G7) gene and cardiovascular disease in case-control and family datasets. Hum. Mol. Genet. 17, 1318-1328. doi: 10.1093/hmg/ ddn 020

van der Harst, P., and Verweij, N. (2018). Identification of 64 novel genetic loci provides an expanded view on the genetic architecture of coronary artery disease. Circ. Res. 122, 433-443. doi: 10.1161/CIRCRESAHA.117.31 2086

Virani, S. S., Alonso, A., Benjamin, E. J., Bittencourt, M. S., Callaway, C. W., Carson, A. P., et al. (2020). Heart disease and stroke statistics-2020 update: a report from the American Heart Association. Circulation 141, e139-e596. doi: 10.1161/CIR.0000000000000746

Wang, D.-C., Wang, H.-F., and Yuan, Z.-N. (2013). Runx2 induces bone osteolysis by transcriptional suppression of TSSC1. Biochem. Biophys Res. Commun. 438, 635-639. doi: 10.1016/j.bbrc.2013.07.131

Wang, L., Hauser, E. R., Shah, S. H., Pericak-Vance, M. A., Haynes, C., Crosslin, D., et al. (2007). Peakwide mapping on chromosome 3q13 identifies the kalirin gene as a novel candidate gene for coronary artery disease. Am. J. Hum. Genet. 80, 650-663. doi: 10.1086/512981

Wienke, A., Herskind, A. M., Christensen, K., Skytthe, A., and Yashin, A. I. (2005). The heritability of CHD mortality in danish twins after controlling for smoking and BMI. Twin Res. Hum. Genet. 8, 53-59. doi: 10.1375/twin. 8.1 .53

Willer, C. J., Li, Y., and Abecasis, G. R. (2010). METAL: fast and efficient metaanalysis of genomewide association scans. Bioinformatics 26, 2190-2191. doi: 10.1093/bioinformatics/btq340

Zdravkovic, S., Wienke, A., Pedersen, N., Marenberg, M., Yashin, A., and De Faire, U. (2002). Heritability of death from coronary heart disease: a 36-year followup of 20966 Swedish twins. J. Intern. Med. 252, 247-254. doi: 10.1046/j.13652796.2002.01029.x 
Zenin, A., Tsepilov, Y., Sharapov, S., Getmantsev, E., Menshikov, L. I., Fedichev, P. O., et al. (2019). Identification of 12 genetic loci associated with human healthspan. Commun. Biol. 2:41. doi: 10.1038/s42003-019-0290-0

Zhang, T., Shen, Y., Chen, Y., Hsieh, J.-T., and Kong, Z. (2015). The ATM inhibitor KU55933 sensitizes radioresistant bladder cancer cells with DAB2IP gene defect. Int. J. Radiat. Biol. 91, 368-378. doi: 10.3109/09553002.2015.10 01531

Zhou, J., Ning, Z., Wang, B., Yun, E., Zhang, T., Pong, R.-C., et al. (2015). DAB2IP loss confers the resistance of prostate cancer to androgen deprivation therapy through activating STAT3 and inhibiting apoptosis. Cell Death Dis. 6:e1955. doi: 10.1038/cddis.2015.289
Conflict of Interest: The authors declare that the research was conducted in the absence of any commercial or financial relationships that could be construed as a potential conflict of interest.

Copyright (c) 2021 Dungan, Qin, Hurdle, Haynes, Hauser and Kraus. This is an open-access article distributed under the terms of the Creative Commons Attribution License (CC BY). The use, distribution or reproduction in other forums is permitted, provided the original author(s) and the copyright owner(s) are credited and that the original publication in this journal is cited, in accordance with accepted academic practice. No use, distribution or reproduction is permitted which does not comply with these terms. 